\title{
Discours
}

Revue de linguistique, psycholinguistique et

informatique. A journal of linguistics, psycholinguistics

and computational linguistics

$16 \mid 2015$

Varia

\section{Anaphore possessive et anaphore associative : le cas des noms collectifs}

\section{Mathilde Salles}

\section{OpenEdition \\ Journals}

Édition électronique

URL : http://journals.openedition.org/discours/8981

DOI : 10.4000/discours.8981

ISSN : 1963-1723

Éditeur :

Laboratoire LATTICE, Presses universitaires de Caen

\section{Référence électronique}

Mathilde Salles, «Anaphore possessive et anaphore associative : le cas des noms collectifs », Discours [En ligne], 16 | 2015, mis en ligne le 09 septembre 2015, consulté le 19 avril 2019. URL : http:// journals.openedition.org/discours/8981 ; DOI : 10.4000/discours.8981

\section{(c) (i) (3) $\Theta$}

Discours est mis à disposition selon les termes de la licence Creative Commons Attribution - Pas d'Utilisation Commerciale - Pas de Modification 4.0 International. 

Revue de linguistique, psycholinguistique et informatique

\title{
Anaphore possessive et anaphore associative : le cas des noms collectifs
}

\author{
Mathilde Salles \\ Université de Caen Basse-Normandie \\ mathilde.salles@unicaen.fr
}

Mathilde Salles, «Anaphore possessive et anaphore associative: le cas des noms collectifs», Discours [En ligne], 16 | 2015, mis en ligne le 9 septembre 2015. 



\title{
Anaphore possessive et anaphore associative : le cas des noms collectifs
}

\author{
Mathilde Salles \\ Université de Caen Basse-Normandie
}

Cet article est consacré à différentes relations anaphoriques pouvant s'établir entre un nom collectif (ex. régiment, caravane, forêt) et les noms de ses membres (ex. soldats, chameaux, arbres, pour les trois noms collectifs précédents): anaphore associative dans des séquences telles que un régiment... les soldats, anaphore possessive de la collection aux membres (un régiment... ses soldats) ou des membres à la collection (des soldats... leur régiment), entre autres exemples. Parfois d'une grande souplesse anaphorique, comparée à une relation sémantique proche comme la méronymie (ex. arbre/tronc, voiture/moteur), la relation membre-collection présente aussi des restrictions surprenantes. Cette souplesse comme ces blocages s'expliquent à la fois par les propriétés référentielles des collections (leur pluralité et leur homogénéité internes) et par certaines propriétés sémantiques de la relation membre-collection (notamment le caractère généralement non relationnel ou catégorématique du nom de membre). On s'intéressera plus spécifiquement ici à l'alternance du défini associatif et du possessif devant les noms de membres. L'homogénéité interne qui caractérise les collections expliquera pourquoi l'anaphore associative n'est pas possible avec certains noms, les noms génériques de membres (qui, eux, sont relationnels; ex. membre, élément): ces derniers ne laissent en effet aucune place à la différenciation réclamée par l'anaphore associative et imposent alors l'emploi du possessif. Le caractère généralement non relationnel du nom de membre expliquera pourquoi le possessif ne constitue pas un véritable concurrent au défini associatif dans les autres cas. On soulignera pour finir que, lorsque toutes les conditions sont réunies pour l'emploi de l'un ou l'autre déterminant, le choix référentiel n'est pas sans incidence sur l'interprétation des relations de cohérence.

Mots clés: anaphore associative, anaphore possessive, noms collectifs, noms relationnels, relations de cohérence

This paper is devoted to some anaphoric operations which are specific to the collection-member relation (e.g., regiment/soldiers, caravan/camels, forest/trees). This relation can be extremely flexible from the anaphoric standpoint, compared to closely related semantic relations such as meronymy (e.g., tree/trunk, car/engine), but can also involve surprising impossibilities. This flexibility and these impossibilities are usually caused, as discussed, by the referential properties of collections (their internal plurality and the homogeneity of their members) and by some semantic properties of the collection-member relation (in particular, the generally non relational nature of the member noun). We study more specifically associative anaphora and possessive anaphora with member nouns. The internal homogeneity of collections explains why associative anaphora is impossible with generic member nouns (relational nouns unlike the others; e.g., member, element): these generic nouns do not allow differentiation while associative anaphora requires differentiation. The generally non relational nature of the member noun explains why possessive anaphora and associative anaphora are not real competitors in other cases. Finally, when all conditions are met for the use of either of the anaphoric processes, we elucidate the consequences of referential choice upon textual coherence relations.

Keywords: associative anaphora, possessive anaphora, collective nouns, relational nouns, coherence relations 
Parmi les diverses relations sémantiques susceptibles de donner lieu à une anaphore associative reconnues par Kleiber (2000 et 200Ia), la relation membre-collection, relation entre un nom collectif (ex. régiment) et les noms de ses membres (ex. soldats), est l'une des plus «anaphoriquement» accueillantes. Comme les relations méronymiques (ex. arbre/tronc), les relations fonctionnelles (ex. voiture/conducteur) et certaines relations locatives (ex. village/église), elle permet à la fois l'anaphore associative (exemples [I]) et l'anaphore possessive allant de la collection aux membres (exemples [2]):

[Ia] Le régiment a été défait. Les soldats n'ont pas eu le temps de combattre. (Kleiber, 2000: 57)

[Ib] Une caravane, qui a traversé à gué, nous arrive dans un état invraisemblable; les chameaux, enduits jusqu'aux yeux de boue gluante, sont devenus des monstres informes et squameux; quant aux mules qui les accompagnaient, elles se sont, paraît-il, noyées dans la vase.

(Frantext: Pierre Loti, Vers Ispahan, Paris, R. Laffont, 1991, p. Ior6)

[2a] Le régiment a été défait. Ses soldats n'ont pas eu le temps de combattre.

(Kleiber, 2000: 59)

[2b] Ensuite ce fut pendant cinq jours une étendue monotone, où l'esprit n'avait pour se distraire et rêver que les jeux de la lumière, le bordj où l'on s'arrête afin de changer d'attelage, quelques tentes noires au ras du sol, la caravane qui chemine avec ses chameaux goudronnés, ses ânes, ses petits chevaux [...].

(Frantext: Jérôme et Jean Tharaud, La fête arabe, Paris, Plon, 1922, p. 8)

Mais elle autorise aussi d'autres types d'anaphores, qui, soit lui sont propres, soit sont autorisés par peu d'autres relations associatives. Ainsi, elle est la seule ${ }^{1}$ à autoriser les anaphores pronominales allant là encore de la collection (d'humains) aux membres avec le ils collectif (exemples [3]):

[3a] Pierre a réuni le comité; ils l'ont tout de suite élu président.

(Flaux, I999: 477)

[3b] Ma famille, qui m’a tout passé jusqu'ici, est résolue à ne point me passer ce qu'ils appellent ma folie.

(Georges Duhamel, Le désert de Bièvres, relevé par Lammert, 2005: 246)

1. Quelques relations fonctionnelles, celles qui unissent un lieu à ses occupants, acceptent toutefois, elles aussi, en plus de l'anaphore associative (ex. une ville... les babitants; une entreprise... les salariés), le ils collectif (ex. À Paris, ils roulent comme des fous [Kleiber, 1994: 163] ; Dans cette usine, ils font grève depuis deux semaines). Mais ces exemples mettent en avant une dimension collective du nom de lieu, du lieu comme regroupement d'individus (Lammert [2010] parlerait ici de «facette collective» de noms intrinsèquement non collectifs). Kleiber (1994: 174) intègre d'ailleurs ville dans la même catégorie d'entités collectives que famille, jury, comité, équipe: il s'agit d'entités qui constituent des groupes. Les noms de lieux peuvent aussi, comme l'ont souligné Borillo (1997) et Flaux (1999), devenir des noms collectifs par métonymie (métonymie du lieu pour ses occupants): ex. Tout Paris est dans la rue. 
Elle est aussi à l'origine de nombreux cas de syllepses, plus ou moins bien reçus $^{2}$ (exemples [4]), qui, comme les ils collectifs, témoignent bien de la pluralité interne associée aux noms collectifs:

[4a] Ce groupe utilise beaucoup l'ordinateur qui leur permet d'une part de vérifier les traductions équivalentes dans leur langue par exemple et d'autre part, de travailler en autonomie leur degré de compréhension.

(mémoire master FLE)

[4b] Ah! oui, les Prussiens [...]. Devant chez le docteur, au bord du ruisseau, il y en avait une troupe, qui s'étaient déchaussés et qui s'enveloppaient les talons avec des chemises de femme garnies de dentelle, volées sans doute à la belle Mme Lefèvre [...].

(Frantext: Émile Zola, La débâcle, Paris, Fasquelle, I897, p. I69; cas de syllepse, si l'on estime que l'antécédent du pronom relatif qui est une troupe, qui le précède immédiatement, et non le pronom en complément du nom troupe)

La relation membre-collection permet enfin l'anaphore possessive allant cette fois des membres à la collection (exemples [5]), ordre anaphorique inversé qu'on ne retrouve qu'avec les relations fonctionnelles (un conducteur... sa voiture) et quelques relations actancielles (un meurtrier... son meurtre vs *une arme... son meurtre):

[5a] Les soldats ont refusé de combattre. Leur régiment a été ainsi défait sans peine.

(Kleiber, 2000: 59)

[5b] [...] une dame du coin nous raconta comment un chameau égaré loin de sa caravane africaine était arrivé là [...].

(Google Books: Alix de Saint-André, En avant, route!, Paris, Gallimard, 20Iо)

Mais cette diversité anaphorique est limitée à certains couples membre-collection et d'autres, comme l'a noté Kleiber (2000), ne permettent plus le possessif devant le nom de membre (exemples [6]) ou devant le nom collectif (exemple [7]):

[6a] Dans les familles d'origine immigrée notamment, la mère/*leur mère [= des familles] est en porte à faux entre sa culture d'origine et sa volonté d'intégration, elle est complètement larguée au niveau scolaire et les enfants/*leurs enfants [= des familles] en profitent.

(Dernières nouvelles d'Alsace du I8 janvier 1998 pour la version définie; Kleiber, 2000 : 57 et 59, 2008: 320)

[6b] Un couple m'a rendu visite hier. Le mari/*Son mari [= du couple] était insupportable. (Milner, 1982: 28, pour la version définie)

2. Reichler-Béguelin (I988: 29) souligne, à propos de ce qu'elle nomme des «anaphores sur syllepse» que «le crédit dont bénéficie le scripteur n'est probablement pas sans influence sur la façon dont sont reçus, par la norme sociolinguistique, ces différents exemples». 
[7] Les arbres resplendissaient de lumière verte. *Leur forêt [= des arbres] était magnifique.

(Kleiber, 2000: 59)

Enfin, devant les noms génériques de membres (membres, éléments), c'est cette fois l'article défini qui paraît difficile et le possessif qui s'impose:

[8a] Le jury a enfin fini de délibérer. Les jurés/?Les membres/Ses membres viennent de regagner leurs sièges.

[8b] J'ai évoqué les Dalfon, cette famille lointaine, et dit combien les capacités de ses membres / ?des membres, dans des domaines multiples, me paniquaient.

(Frantext: Albert Simonin, Confessions d'un enfant de La Chapelle, Paris, Gallimard, 1984, p. 44)

Si Kleiber (2000) a expliqué les différences entre [2] et [6] et entre [5] et [7], c'est-à-dire entre les noms de membres qui autorisent le possessif et ceux qui ne l'autorisent pas d'une part, et entre les noms de collection qui autorisent le possessif et ceux qui ne l'autorisent pas d'autre part, le cas illustré par l'exemple [8] n'a, à ma connaissance, jamais reçu d'explication. Après avoir présenté les analyses de Kleiber pour rendre compte de la distribution du possessif dans les premiers cas, on s'attachera donc aux cas où le défini associatif ne convient plus, c'est-à-dire aux cas où l'on n'a plus affaire à un nom spécifique de membre, mais à un nom générique. Ces cas, comme on le verra, s'expliquent par un des critères généralement avancés pour définir les collectifs, celui de leur homogénéité interne ${ }^{3}$. Mais cette explication remet alors en question le critère de l'homogénéité pour d'autres emplois anaphoriques des noms collectifs.

On s'intéressera, pour finir, aux cas où la concurrence entre possessif et défini associatif est a priori possible (voir les exemples [I]-[2]). Les véritables cas de concurrence se révéleront finalement plus rares que pour d'autres relations associatives, la différence tenant au caractère généralement non relationnel (ou catégorématique) du nom de membre.

L'étude sera ici limitée aux noms collectifs les plus prototypiques, les noms «collectifs de groupe» de Leisi (1971) - cité par Mihatsch (2000) - comme régiment, famille, forêt, troupeau, désignant des collections fondées sur un regroupement spatio-temporel, fonctionnel ou social de leurs membres (voir Lammert, 2005 et 2010).

3. Outre la définition des noms collectifs proposée par Flaux (I999: 472; je souligne): «noms dénotant des entités composées de parties distinctes préalablement constituées, homogènes entre elles, mais autonomes et hétérogènes par rapport au tout», voir entre autres, Michaux (I992), Borillo (I997), Flaux (1998), Lecolle (I998), Flaux et Van de Velde (2000), Kleiber (200Ib), Lammert (2005, 2006 et 20I0). 


\section{L'emploi du possessif ou la double source de Kleiber (2000 et 2004)}

La possibilité ou non d'avoir le possessif devant un nom de membre (exemples [2] et [6]) ou un nom collectif (exemples [5] et [7]) s'explique, selon Kleiber (2000), soit par les propriétés ontologiques des entités désignées, soit par le type de relation sémantique entre les unités lexicales. Kleiber (2000 et 2004) pose en effet l'hypothèse d'une double source pouvant fournir au possessif la relation de dépendance qu'il exige.

I) Celle-ci peut lui être fournie par le statut ontologique des entités désignées, selon l'échelle de dépendance ontologique suivante (Kleiber, 2000: 69):

humains $>$ animaux $>$ objets concrets $>$ événements $>$ propriétés

Cette hiérarchie complète celle de Hawkins (198I), qui n'ordonnait que les humains (et leurs parties du corps), les animés non humains et les inanimés, pour décrire les constructions possessives anglaises $N P^{\prime} s N$ et the $N$ of $N P^{4}$. Si l'échelle proposée par Kleiber (2000) ne mentionne pas explicitement les collections, il semble toutefois considérer que, lorsque celles-ci sont des collections d'inanimés, elles se situent au même niveau que les objets concrets (voir à cet égard, ses remarques sur l'identité de niveau ontologique de forêt et arbres, p. 7I) et que, lorsqu'il s'agit de collections d'animés humains, elles sont d'un niveau inférieur aux humains (voir, cette fois, ses remarques sur la possibilité d'avoir son régiment pour le régiment de Bernard, ou leur régiment pour le régiment des appelés d'outre-mer, p. 70).

En français, l'emploi du déterminant possessif serait donc approprié lorsque l'entité «possesseur» est ontologiquement supérieure à l'entité «possédée». C'est le cas, si l'on admet les positions ontologiques qu'on vient d'évoquer, en [5] avec les $\mathrm{SN}$ possessifs leur régiment, sa caravane: l'ordre de dépendance ontologique est respecté, le possédé, collection, étant ontologiquement inférieur au(x) possesseur(s), humains (les soldats pour le régiment) ou animal (le chameau pour la caravane). Ce n'est plus le cas, en revanche, ni en [6], avec les SN leur mère, leurs enfants (= des familles) et son mari (= du couple), ni en [7], avec le SN leur forêt (= des arbres): en [6], le possédé, humain, est d'un statut ontologique supérieur au possesseur, collection; en [7], les statuts ontologiques possédé-possesseur, forêt-arbres, sont identiques, ce qui empêche aussi la détermination possessive.

La hiérarchie ontologique n'est cependant pas toujours respectée, puisqu'on trouve aussi, ses soldats (= du régiment), ses chameaux (= de la caravane) en [2], autrement dit des «possédés» humains ou animaux avec des «possesseurs» situés à un niveau inférieur de l'échelle, ou encore ses arbres, pour «les arbres de la forêt»,

4. Selon Hawkins, cette hiérarchie détermine l'ordre d'occurrence des noms dans ces constructions, les noms de possesseurs humains précédant les noms de possesseurs animés non humains, eux-mêmes précédant les noms de possesseurs non animés. Cela expliquerait le choix de Mary's cat/car et non de ?the cat/car of Mary, de the cat's basket et non ?the basket of the cat, etc. (ces exemples sont repris à Hawkins, I98r : 257 et 260). 
avec un possesseur, la forêt, de statut ontologique égal. Mais, on l'a dit, la source de dépendance est double chez Kleiber ${ }^{5}$, et, si la hiérarchie ontologique ne fournit pas l'ordre de dépendance favorable au possessif, celui-ci peut lui être fourni par la relation sémantique en jeu.

2) «Si deux entités sont du même type ou si une entité d'un type inférieur se présente comme l'antécédent d'une entité de type supérieur, c'est alors une relation lexicale qui prend le relais et qui peut fournir l'asymétrie déterminative nécessaire a priori» (Kleiber, 2000: 7I) ${ }^{6}$.

La relation membre-collection fournit bien une telle asymétrie déterminative: si le membre, entité autonome, n'est pas défini par sa relation, contingente, à la collection, la collection, elle, est définie par rapport aux éléments qui la composent. Comme il a souvent été noté ${ }^{7}$, cette orientation de la collection aux membres différencie d'ailleurs fondamentalement la relation membre-collection de la relation partie-tout au sens strict. Il y a asymétrie dans les deux cas, mais l'orientation ne se fait pas dans le même sens, de la collection vers les membres, dans un cas, de la partie vers le tout, dans l'autre: ainsi, alors qu'un arbre n'est défini ni par ses parties, ni par son éventuelle appartenance à un ensemble plus vaste, les parties de l'arbre seront, elles, définies comme telles (un tronc se définit comme une partie d'un arbre) et une forêt sera définie comme un ensemble d'arbres. Mais, en dépit de ces différences d'orientation, la relation possessive va toujours de l'entité englobante à l'entité englobée: ses arbres (= de la forêt; une forêt a/possède des arbres) et non *leur forêt (= des arbres; ${ }^{*}$ des arbres ont/possèdent une forêt), son tronc (= de l'arbre; un arbre a/possède un tronc) et non ${ }^{*}$ son arbre (= du tronc; ${ }^{*}$ un tronc alpossède un arbre).

La relation membre-collection offre donc, selon Kleiber (2000), l'orientation dépendancielle favorable au possessif dans ses soldats (= du régiment; voir un régiment a/comporte des soldats, Kleiber, 2000: 72), ses chameaux (= de la caravane) ou encore ses arbres (= de la forêt; voir une forêt a/possède/comporte des arbres, Kleiber, 2000: 7I), avec des possesseurs de statut ontologique inférieur ou égal au possédé.

Reste à expliquer l'impossibilité d'avoir le possessif en [6], devant des noms de parenté comme mère ou mari, après les noms collectifs famille ou couple. On a déjà souligné que ces formations contrevenaient à la hiérarchie ontologique. La relation membre-collection qui permet des anaphores associatives telles que une famille... la mère, un couple... le mari, ne parait pas offrir, elle non plus, l'orientation dépendancielle réclamée par le possessif. Selon Kleiber (2000: 72 ), si le possessif ne convient pas devant les noms mari et mère, c'est parce que «la relation fonctionnelle qui les caractérise sémantiquement les unit non pas à famille ou couple, mais à enfants pour mère et femme

5. Elle sera triple dans des écrits postérieurs (Kleiber, 2008, 2009 et 20II) avec une source discursive que je n'évoquerai pas ici.

6. Kleiber (2000: 70 , notes 25 et 26 ) souligne lui-même que son hypothèse explicative ne règle pas tous les cas (ex. ma petite, mon salaud, mon colonel...).

7. Voir notamment Lecolle (1998), Villar (2006) et Joosten (2010). 
pour mari». Le possessif activerait ainsi un mode de saisie référentielle différent, fondé sur la parenté directe entre individus (voir Kleiber, 2003). Ce qui n'est plus le cas évidemment dès qu'on n'a plus affaire à des noms de parenté comme mari ou mère, mais à un strict nom de membre, le nom membre. Cette fois, la relation membrecollection retrouve droit de cité et offre au possessif (ses membres pour les membres de la famille; voir Kleiber, 2000: 72) l'orientation dépendancielle qu'il réclame (une famille a/comporte des membres). Mais alors, on l'a déjà noté avec les exemples [8] repris ici en [9], c'est le défini qui ne convient plus, et cela après n'importe quel nom collectif:

[9a] Le jury a enfin fini de délibérer. Les jurés/ ?Les membres/Ses membres viennent de regagner leurs sièges.

[9b] J'ai évoqué les Dalfon, cette famille lointaine, et dit combien les capacités de ses membres/ ?des membres, dans des domaines multiples, me paniquaient.

(Frantext: Albert Simonin, Confessions d'un enfant de La Chapelle, Paris, Gallimard, I984, p. 44)

\section{Les noms génériques de membres}

Ce jugement concernant l'anaphore associative avec un nom générique comme membre ne fait toutefois pas l'unanimité. Lammert (2010: IIO) propose ainsi l'exemple (construit) d'anaphore associative suivant:

[Io] Le comité ne rentrera pas de Brest ce soir, les membres resteront sur place une nuit de plus.

(Lammert, 20I0: IIо)

Cette séquence paraît pourtant difficile, comparée à la version possessive donnée en $[\mathrm{II}]$ :

[II] Le comité ne rentrera pas de Brest ce soir, ses membres resteront sur place une nuit de plus.

Une recherche de cooccurrences sur Frantext ${ }^{8}$ confirme indirectement cette difficulté: à une exception près (qui, on le verra, n'en est pas vraiment une), aucune des 37 séquences les membres relevées après une occurrence de comité, ne s’interprète comme un anaphorique associatif de ce dernier. Le caractère défini de la séquence les membres provient toujours d'une autre source; c'est très majoritairement (35 cas

8. Recherche effectuée sur Frantext intégral (4515 textes) avec la demande de cooccurrence suivante: comité précédant les membres, "pas nécessairement dans la même phrase», en utilisant la valeur de distance maximale par défaut ( 20 mots). Cette recherche fournit 37 résultats. Une recherche comparable avec le possessif (cooccurrence comitélses membres) fournira 32 résultats. 
sur 37) un complément du nom membres qui permet le défini : complément en de + SN le plus souvent (exemple [12]), pronom relatif dont (exemple [13]), pronom en (exemple [I4]), relative restrictive (exemple [I5]):

[I2] Le bill de M. Fox était une véritable saisie judiciaire; il retirait à la compagnie toutes ses propriétés, et les plaçait en régie entre les mains d'un comité chargé de gérer pour elle, de liquider ses dettes, et de disposer de tous les emplois. Les membres du comité, nommés par le roi ou le parlement, devraient être inamovibles, et siéger jusqu'à ce qu'ils eussent mis les affaires sur un meilleur pied.

(Frantext: Emmanuel de Las Cases, Mémorial de Sainte-Hélène, Paris, Gallimard, 1956-1957, p. 279)

[13] Ce corps est une sorte de comité chargé de l'administration de la police, et dont les membres sont choisis dans le conseil de ville.

(Frantext: Louis Ramond de Carbonnières, Lettres de M. William Coxe à M. W. Melmoth sur l'état politique, civil et naturel de la Suisse, Paris, Belin, I78I, p. I80)

[14] Va pour l'Odéon («Va pour le champagne, d'Arpentigny!»), mais ce n’est pas assez d'avoir les deux directeurs; il y a un Comité de lecture à l'Odéon, il faut d'avance en connaitre les membres... et qu'on les chauffe.

(Frantext: Gustave Flaubert, Correspondance (1854-1857), Paris, L. Conard, 1927, p. 97)

[I5] Je vous en ai démontré les erreurs pour vous armer de défiance, et non pour me donner le triste plaisir de censurer le travail du comité de marine. J'invite les membres éclairés qui composent ce comité à la plus sérieuse attention.

(Frantext: sans mention d'auteur, Journal de la Société de 1789, Paris, Edhis, 1982, p. I8)

Une des deux occurrences de la séquence les membres qui restent est bien anaphorique mais pas associative (exemple [i6]); elle s'interprète comme une anaphore coréférentielle (le SN les membres s'y interprète comme «les membres des Nations unies» et non comme «les membres du comité»). L'autre occurrence (exemple [17]) semble bien associative (les membres de droit s'y interprète bien comme «les membres de droit de chaque jury ou comité d'examens»), mais on n'a plus affaire au seul SN les membres et avec la spécification de droit, on y reviendra, on n'a plus affaire à la même généricité:

[16] Le comité IV-2 fut très vite d'accord que les membres des Nations unies devaient assumer les mêmes obligations que celles imposées sous le régime de la SDN pour enregistrer les traités et accords internationaux, le secrétariat ayant également charge de les publier. Le terme «accords internationaux» fut employé de préférence à «engagements internationaux», afin de couvrir les entreprises unilatérales de caractère international et ainsi de réserver une place pour l'exemption des engagements 
militaires secrets, que les membres des Nations unies pouvaient prendre avec le conseil de sécurité, ou entre eux, pour le maintien de la paix et de la sécurité, dont le comité avait des raisons de croire que les membres pouvaient ne pas désirer qu'ils fussent publiés.

(Frantext: sans mention d'auteur, La Charte des Nations unies: statut de la Cour internationale de justice: texte de la Déclaration de Potsdam, Paris, Hachette, 1946, p. 56)

[17] décret du 22-2-1948. chaque jury ou comité d'examens est composé de quatre membres au moins et de douze au plus, non compris les membres de droit.

(Frantext: sans mention d'auteur, L'enseignement en France: l'enseignement de la musique et l'éducation musicale. I. L'enseignement officiel, Paris, La Documentation française, 1950, p. I2)

Si l'on ne relève aucune anaphore associative entre un comité et les membres, on relève, en revanche, de nombreuses anaphores possessives d'un comité à ses membres; trois exemples parmi d'autres:

[I8] Je n'ai point dit au comité les motifs de ma démission, non seulement pour conserver en entier le droit de tous les membres de la société, mais encore parce qu'il est des motifs que l'on doit taire. Pour faire comprendre au comité l'imprudence de sa doctrine, qui ne résulte d'aucun article des statuts, car il n'est dit nulle part que le comité sera juge d'une démission, j’invoque le témoignage de deux de ses membres: MM. Pyat et Merruau.

(Frantext: Honoré de Balzac, Correspondance, Paris, Garnier, t. IV, I840-avril 1845, I966, p. 325)

[19] Ce document eut pour résultat de refaire l'unanimité de notre comité de Londres. Tous ses membres virent clairement où était la voie nationale.

(Frantext: Charles de Gaulle, Mémoires de guerre, Paris, Plon, t. II, L'unité (19421944), 1956, p. 98)

[20] [...] j'estimais que la présence de Sartre rendait la mienne superflue. Cependant, connaissant à travers Sartre les décisions du Comité, j’approuvais que ses membres s'engageassent à ne pas écrire dans les revues et les journaux qui accepteraient des textes d'anciens collabos.

(Frantext: Simone de Beauvoir, La force des choses, Paris, Gallimard, 1963, p. 36)

Comment expliquer cette préférence du possessif devant un nom générique de membre? Outre leur généricité, des noms comme membre, élément, constituant présentent une différence fondamentale avec la plupart des autres noms entrant dans la relation membre-collection, différence qui a des incidences importantes sur la relation membre-collection elle-même. En effet, contrairement aux autres (ou à la plupart des autres), ce sont des noms relationnels, autrement dit des noms qui ont, selon Hiz (I968), un complément explicite ou tacite, et qui «implique[nt] une 
mise en relation» (Milner, 1982: 28) ${ }^{9}$; ou encore, selon les termes de Kleiber (198I), des noms syncatégorématiques. Or, les noms de collections sont, eux aussi, syncatégorématiques. C'est dire qu'on ne retrouve plus l'asymétrie censée caractériser la relation membre-collection ${ }^{10}$.

La clef de l'explication ne semble toutefois pas être dans cette double syncatégorématicité. En effet, d'autres noms de membres, non génériques, sont des noms relationnels définis par leur appartenance à une collection ${ }^{11}$ et acceptent parfaitement l'anaphore associative. Il s'agit de noms de membres comme jurés et choristes, formés par dérivation sur les noms collectifs jury et chour. Voir l'exemple proposé en [8a] et [9a], redonné ici en [2I]:

[2I] Le jury a enfin fini de délibérer. Les jurés/?Les membres/Ses membres viennent de regagner leurs sièges.

On peut sans difficulté construire l'anaphore associative le jury... les jurés, alors que la séquence le jury... les membres paraît difficile.

Kleiber (1999: 298, note 24) pose au sujet des noms collectifs et de l'anaphore associative une question extrêmement intéressante: est-il possible d'avoir une anaphore associative avec des noms collectifs homogènes et, à partir du moment où un collectif homogène entre en anaphore associative ne perd-il pas cette homogénéité interne? Si le défini associatif extrait un ou des éléments distincts des autres ou d'autres éléments de l'ensemble, alors l'homogénéité interne constitutive des noms collectifs devrait en effet représenter un sérieux frein à certaines anaphores associatives, celles qui réfèrent à la totalité des membres. On peut rappeler à ce sujet l'analyse de Kleiber (2oora: 80), qui souligne bien les conséquences de la valeur d'unicité du défini pour l'anaphore associative: «l'entité dénotée par l'expression associative est présentée comme étant la seule entité (ou le seul ensemble d'entités en cas d'article défini pluriel) de la catégorie $\mathrm{N}$ à l'intérieur du cadre que constitue l'entité dénotée par l'antécédent», cette dernière entité devant alors contenir «d'autres entités que l'entité de l'expression anaphorique».

Le problème ne se pose pas avec des exemples tels que une famille... le père... le fils, un couple... le mari, ou encore une caravane... les chameaux, une forêt... les chênes... les hêtres..., etc.: les SN définis, en spécifiant des types de membres, ne supposent pas une référence à l'ensemble des membres, ils ont bien une

9. Voir aussi Barker (20II : IIO9): "In contrast with a sortal noun (e.g., person), friend is a (two-place) RELATIONAL NOUN: a person counts as a friend only in virtue of standing in a particular relationship with another individual»; ou encore Herslund (1996), qui cite d'ailleurs membre et medlem («membre (d'une association)», en danois) comme exemples de noms relationnels.

10. On a rappelé plus haut son orientation de la collection vers les membres, opposée à l'orientation de la partie vers le tout, qui caractérise la relation partie-tout au sens strict. Alors que, dans une relation partie-tout, c'est le nom de partie qui est syncatégorématique, dans une relation membre-collection, c'est le nom de collection qui l'est.

11. On ne parle pas ici des noms relationnels de parenté évoqués dans la section I. 
valeur d'extraction. Mais que dire de séquences comme une forêt... les arbres, un régiment... les soldats, un jury... les jurés?

Selon Lammert (2005, 2006, 2010), les collections, notamment les collections inanimées comme une forêt ou un bouquet, présentent malgré tout une certaine hétérogénéité: à côté d'une série de membres principaux homogènes (respectivement des arbres et des fleurs), ces collections peuvent inclure des éléments secondaires (fougères, champignons, etc. pour une forêt; branchages, éléments de verdure pour un bouquet). C'est-à-dire que sans renoncer au principe définitoire de l'homogénéité, Lammert la restreint en quelque sorte à des éléments principaux obligatoires, une forme d'hétérogénéité pouvant être apportée par des éléments facultatifs. Il n'y aurait plus d'obstacle alors à l'établissement d'une anaphore associative comme une forêt... les arbres, puisque les arbres, éléments principaux, ne sont pas les seuls éléments constitutifs de la forêt; l'anaphore associative peut alors se poursuivre en énumérant d'autres éléments: une forêt... les arbres... les ronces... les fougères... On pourrait tenir un raisonnement proche pour expliquer des séquences comme un régiment... les soldats, un orchestre... les musiciens: outre les membres constitutifs obligatoires, il peut exister des éléments "satellites», membres de la collection comme les chevaux d'un régiment de cavalerie, ou individus dotés d'un rôle fonctionnel, comme le colonel et le chef d'orchestre, sous la direction desquels sont placés le régiment et l'orchestre ${ }^{12}$. Il peut aussi y avoir une certaine hiérarchisation entre les membres permettant des distinctions parmi un ensemble: dans un jury, il peut y avoir un membre président ou un premier juré, des jurés titulaires ou suppléants.

Le problème des noms génériques de membres en anaphore associative est lié à un effet d'homogénéisation justement, d'indifférenciation de ces membres. Avec des SN comme les membres, les éléments, la référence se fait de façon indifférenciée à l'ensemble des membres et l'homogénéité de la collection est mise en avant. La moindre différenciation, par exemple au moyen de l'adjonction de l'élément prépositionnel de droit dans l'exemple [17], repris ici en [22], ou de l'adjectif titulaires au nom membres en [23], rendra de nouveau l'anaphore associative possible:

[22] décret du 22-2-1948. chaque jury ou comité d'examens est composé de quatre membres au moins et de douze au plus, non compris les membres de droit.

(Frantext: sans mention d'auteur, L'enseignement en France: l'enseignement de la musique et l'éducation musicale. I. L'enseignement officiel, Paris, La Documentation française, 1950, p. I2)

12. La relation sémantique est ici plutôt une relation fonctionnelle au sens de Kleiber (2oora) qu'une stricte relation membre-collection: le colonel et le chef d'orchestre exercent une fonction vis-à-vis du régiment et de l'orchestre (voir aussi Lecolle [1998: 5I], qui compare les relations «membre fonctionnelcollection» - chef d'orchestre/orchestre, père/famille - aux relations "partie fonctionnelle-objet»). Si la relation fonctionnelle l'emporte, la structure qui s'applique habituellement aux membres de collections «un Ncoll de (nombre) N de membres» ne convient plus: un orchestre de Ioo musiciens, un orchestre de violonistes, mais ?un orchestre de roo musiciens et un chef. 
[23] Les nouveaux venus du Politburo sont des Russes, en ce qui concerne les membres titulaires (Romanov, Premier secrétaire du comité régional de Leningrad et le maréchal Oustinov, ministre de la Défense) et l'unique nouveau venu parmi les suppléants, Aliev, vient en remplacement d'un dirigeant national déchu de ses fonctions, Mjavanadzé.

(Frantext: Hélène Carrère d'Encausse, L'Empire éclaté: la révolte des nations en URSS, Paris, Flammarion, 1992, p. 172) (Kleiber, 2009: 30I) en position d'objet:

Mais lorsque la collection est présentée comme totalement homogène avec des noms de membres génériques, le défini associatif est exclu. Pour autoriser l'anaphore associative, les noms collectifs doivent ou perdre une part de leur homogénéité interne en admettant des différenciations entre les membres, ou pouvoir être associés à des éléments fonctionnels externes (par exemple, le berger pour un troupeau) grâce à d'autres relations sémantiques «associatives», les relations fonctionnelles. Alors que ses $N$ permet l'homogénéisation des membres, les $N$ suppose une forme de différenciation.

\section{La concurrence le $\mathrm{N} / \operatorname{son} \mathrm{N}$}

Il reste à expliquer dans quels cas, lorsque la concurrence est a priori possible, on préférera l'anaphore associative (ex. un régiment... les soldats, une caravane... les chameaux) à l'anaphore possessive (ex. un régiment... ses soldats, une caravane... ses chameaux), et inversement. Des éléments de réponse, liés à la différence de saisie référentielle (contraste interne à l'entité antécédent pour le défini associatif, en l'occurrence contraste avec d'autres éléments de la collection, ou contraste avec d'autres collections pour le possessif: ses soldats, dirait Kleiber, ouvre le paradigme des soldats appartenant à d'autres entités, ceux du régiment $\mathrm{X}, \mathrm{Y}$ ou $\mathrm{Z}$ ) et de continuité topicale ont déjà été apportés par Kleiber (2004, 2008, 2009 et 20II):

Le possessif construit une chaîne topicale différente: l'antécédent reste saillant au niveau du nouveau référent de l'expression possessive par l'intermédiaire de l'adjectif possessif. L'identification de ce référent ne se réalise plus dans un ensemble englobant contenant d'autres entités de même niveau [comme c'est le cas avec le défini associatif], mais provient d'une mise en relation directe avec l'antécédent.

Outre cette différence de contraste, le déterminant possessif maintient, à cause de sa part pronominale, une certaine continuité topicale et réclame, selon Kleiber (2009: 30I), que «l'antécédent soit dans une certaine position de saillance».

Les deux exemples suivants constituent une bonne illustration des différences mises en avant par Kleiber - contraste avec un autre bouquet en [24] et continuité topicale avec reprise par le possessif du sujet (II) de la phrase précédente; anaphore associative en [25], où il n'est pas question d'un autre bouquet, avec un antécédent 
[24] Reprenez votre microscope, et considérez cet autre bouquet. Il n'est pas fait précisément comme le précédent. Ses fleurs sont aussi en cloches.

(Frantext: Charles Bonnet, Contemplation de la nature, Neuchâtel, S. Fauche, I78I, p. 327)

[25] Enfin, après quelques minutes d'hésitation, elle prit le bouquet, et, en touchant les fleurs dans l'obscurité profonde, elle sentit qu'un billet était attaché à la tige d'une fleur $[\ldots]$.

(Frantext: Stendhal, L'abbesse de Castro, Paris, A. Colin, I960, p. I53)

Mais, avec les noms collectifs, la conséquence la plus importante de la part pronominale du possessif, même si elle n'est pas indifférente au statut topical de l'antécédent, est finalement bien plus triviale: le possessif est majoritairement utilisé pour anaphoriser des antécédents humains, suivant la "pronominalization propensity» de Fraurud (1992 et 1996: 67), «showing that buman referents are more often referred to by pronouns than non-buman referents». Nos recherches sur Frantext l'illustrent bien: lorsqu'on effectue des demandes de cooccurrences entre un nom collectif et un SN possessif référant à ses membres (ex. classe/ses élèves, régiment ou division/ses soldats, équipage/ses marins, troupeau/ses moutons, etc.), les résultats obtenus, lorsqu'il y en a, ne sont presque jamais des anaphores possessives comme celle de l'exemple [24], mais presque toujours des anaphores possessives se rattachant à un possesseur humain ${ }^{13}$ comme en [26] et [27]:

[26] C'était le maitre d'école, un fils de paysan, qui avait sucé la haine de sa classe avec l'instruction. Il violentait ses élèves, les traitait de brutes et cachait des idées avancées, sous sa raideur correcte à l'égard du curé et du maire.

(Frantext: Émile Zola, La terre, Paris, Bernouard, 1929, p. 56)

[27] Vous voyez bien ce colonel qui est là devant le grand perron, avec son régiment en bataille. Nous irons à lui, et là, devant ses soldats, je le sommerai de se ranger au devoir, et de rendre à la république son régiment.

(Frantext: Victor Hugo, Histoire d'un crime. Déposition d'un témoin, Paris, R. Laffont, 1987, p. 403)

La concurrence entre possessif et défini associatif est donc possible a priori, mais l'anaphore associative se révèle bien plus productive. Et le possessif est bien moins utilisé et bien moins concurrentiel qu'avec les relations fonctionnelles (exemples [35]-[38]) ou les relations méronymiques (exemples [39]-[40]):

[35] Fauillet (47): une voiture heurte un arbre, son conducteur décède.

(Sud Ouest du 5 janvier 2014; en ligne: http://www.sudouest.fr/20r4/or/o5/fauillet47-une-voiture-heurte-un-arbre-son-conducteur-decede-I420I05-3755.php)

13. Humain qui est aussi, en [26] et [27], le «possesseur» de la collection (sa classe, son régiment). 
[36] Ivre, elle détériore une voiture et griffe le conducteur à Monchecourt. (La voix $d u$ Nord du II janvier 20I4; en ligne: http://www.lavoixdunord.fr/region/ ivre-elle-deteriore-une-voiture-et-griffe-le-conducteur-iaı6bonı838563)

[37] Pas du tout, ma belle dame, je n'admire point le roman de Mlle Bosquet: une femme bien élevée, qui est un livre absolument raté, comme j'ai eu l'honneur de le dire à son auteur.

(Frantext: Gustave Flaubert, Correspondance (1862-1865), Paris, L. Conard, I929, p. I68)

[38] Quant à moi, ne crois pas que ce livre m'ait irrité (il y a longtemps que je sais à quoi m'en tenir sur l'auteur!).

(Frantext: Gustave Flaubert, Correspondance: supplément (I858-1860), Paris, L. Conard, I954, p. 253)

[39] L'arbre résista; son tronc puissant bien qu'entaillé jusqu'au milieu, était rigide comme du fer.

(Frantext: Guy de Maupassant, Contes et nouvelles, Paris, A. Michel, t. II, 1885, 1960, p. I036)

[40] Et, serrant sa jupe, la rattachant par-devant à sa ceinture, sans voir qu'elle montrait ses cuisses, elle prit l'arbre nerveusement, se hissa sur le tronc, d'un seul effort des poignets.

(Frantext: Émile Zola, La faute de l'abbé Mouret, Paris, Gallimard, 1960, p. 1364)

En fait, c'est avec des noms relationnels que le possessif apparait comme un véritable concurrent du défini associatif. Leur caractère relationnel leur permet d'échapper à l'effet de "pronominalization propensity» humaine observé ailleurs. La dépendance relationnelle, relation partie-de ou relation fonctionnelle, l'emporte aisément sur des relations de dépendance contingentes. C'est-à-dire que face à un SN de la forme «son + N relationnel», son tronc, son conducteur, son auteur, même si des interprétations discursives ou pragmatiques (voir Bartning, 1996; des interprétations liées à des informations contextuelles ou des connaissances extralinguistiques particulières, par exemple, son tronc, le tronc de Max = celui auquel il applique de la bouillie bordelaise, celui contre lequel il a l'habitude de s'adosser, etc.) sont possibles, l'interprétation relationnelle, prototypique (voir là aussi Bartning, 1996), est prégnante.

Le caractère généralement catégorématique, c'est-à-dire non relationnel, des noms de membres explique ainsi pourquoi ils sont rarement déterminés par le possessif lorsqu'il s'agit d'exprimer la relation de dépendance à la collection ${ }^{14}$ et

14. Une recherche dans la base Frantext non catégorisée (recherche effectuée dans les textes postérieurs à 1900) souligne qu'avec d'autres noms catégorématiques, les noms des entités incluses (ex. église/village, réfrigérateur/cuisine) des relations locatives de Kleiber, le possessif est aussi plus souvent utilisé pour 
pourquoi il n'y a pas de véritable concurrence entre anaphore possessive et anaphore associative. Il n'y a pas non plus de concurrence entre possessif et défini avec les noms de membres relationnels puisque, parmi ceux-ci, les noms génériques n'autorisent que le possessif (un comité... ses membres/?les membres), les noms de parenté n'autorisent que le défini (un couple... le mari/*son mari) et les noms formés par dérivation sur le nom collectif comme jurés, choristes, s'accommodent assez mal du possessif, souvent un peu redondant (Le jury a enfin fini de délibérer. ?Ses jurés viennent de regagner leurs sièges. Voir toutefois l'exemple suivant relevé sur le site d'information de rtbf.be [26/11/2013] : Pour l'actuel procès qui se tient à Mons concernant l'assassinat d'Albana Margjeka, en janvier 2008, le jury est composé de cinq femmes et de sept hommes. Or, un de ses jurés a interpellé la cour ce lundi matin: durant le week-end, a-t-il raconté, il a été contacté par un ami d'un des deux accusés, la compagne de la victime à l'époque, qui aurait tenté de l'influencer dans son jugement sur l'affaire [https://www.rtbf.be/info/regions/detail_assises-de-mons-un-des-juresa-subi-une-forme-de-pression-un-cas-rarissime?id=8143707]). Mais, si l'on étend la relation membre-collection à un couple comme ville/babitants (voir note I), avec le nom relationnel babitants, on observe enfin une véritable concurrence entre anaphore possessive (exemples [4I]-[44]) et anaphore associative (exemples [45]-[48]):

[4I] Les magistrats des amycléens, fatigués des fausses nouvelles qui plusieurs fois avoient menacé leur ville d'un siege, défendirent sous peine de mort qu'on en parlât davantage. En conséquence du silence imposé par cette loi, les ennemis vinrent tout de bon, la ville fut prise et ses habitants furent égorgés; il ne se trouva pas de citoyen assez généreux pour avertir sa patrie du péril auquel elle se trouvoit exposée; un amycléen courageux eût-il donc été coupable si, méprisant une loi extravagante, il eût annoncé hardiment une vérité dangereuse, mais nécessaire au salut de tous ses concitoyens?

(Frantext: Paul Henri d'Holbach, La morale universelle ou les devoirs de l'homme fondés sur la nature, Amsterdam, M.-M. Rey, 1776, p. 135)

[42] M. Martini tira de sa poche le guide Sarty et lut: «Cette ville fut à son origine une colonie fondée par les Phocéens de Marseille, vers l'an 340 avant J.-C. Elle reçut d'eux le nom grec d'Antipolis, c'est-à-dire "contre-ville", ville en face d'une autre, parce qu'en effet elle se trouve opposée à Nice, autre colonie marseillaise.

Après la conquête des gaules, les romains firent d'Antibes une ville municipale; ses habitants jouissaient du droit de cité romaine. [...]»

(Frantext: Guy de Maupassant, Contes et nouvelles, Paris, A. Michel, t. I, I886, 1959, p. 729)

référer à un humain que pour référer à l'entité englobante: les occurrences de son église avec interprétation locative («église du village/de Saint-Nectaire, etc.») sont en effet bien moins fréquentes (44 occurrences sur 205) que les interprétations à possesseur humain ou divin («église du curé/de Jésus/de Dieu/de l'évêque/de Mme Plutarque, etc.»). Et, parmi les 19 occurrences de son réfrigérateur, son frigo ou son frigidaire relevées, une seule reçoit une interprétation locative (son frigidaire comme «le frigidaire de la cuisine»), les I8 autres étant rattachées à un possesseur animé. 
[43] L'ancienne malédiction sort du pays, où il n'y aura plus désormais de voleur ni de parjure. Les jours de jeûne, institués pour expier les fautes passées, doivent être changés en joyeux jours de fête. Jahvé aime Sion d'une passion ardente, et il en fera une ville fidèle, une montagne sainte. Ses habitants seront son peuple, et il sera leur dieu en vérité et en justice. Eux, qui avaient été une malédiction parmi les nations, seront une bénédiction; mais ils devront pratiquer la sincérité et la justice, car Jahvé hait le faux serment.

(Frantext: Dictionnaire de théologie catholique, A. Vacant et E. Mangenot (dir.), Paris, Letouzey et Ané, t. IV, I920, p. I003)

[44] L’Inspecteur. - C’est peu. Et qu'avez-vous rêvé, cette nuit, mon cher maire?/Le Maire. - Ce que j'ai rêvé, pourquoi?/L'Inspecteur. - Si l'atmosphère de la ville est à ce point purifiée, ses habitants doivent jouir des rêves les plus normaux de France. Vous rappelez-vous ce que vous avez rêvé?

(Frantext: Jean Giraudoux, Intermezzo, Paris, B. Grasset, 1933, p. 97)

[45] Le porteballe détermina, par l'espoir d'une récompense, les paysans à courir après Jacques. Voilà donc une multitude d'hommes, de femmes et d'enfants allant et criant: «au voleur! Au voleur! À l'assassin!» et le porteballe les suivant d'aussi près que le fardeau dont il était chargé le lui permettait, et criant: «au voleur! Au voleur! À l'assassin! ...»

Ils sont entrés dans la ville, car c'est dans une ville que Jacques et son maître avaient séjourné la veille; je me le rappelle à l'instant. Les habitants quittent leurs maisons, se joignent aux paysans et au porteballe, tous vont criant à l'unisson: «au voleur! Au voleur! À l'assassin! ...»

(Frantext: Denis Diderot, Jacques le Fataliste et son maître, Paris, Garnier, 1965 , p. 518 )

[46] Kennedy ne répondit pas; il préférait se taire à discuter cette terrible possibilité. Le docteur signala la ville de Lari vers les cinq heures du soir. Les habitants travaillaient à la récolte du coton devant des cabanes de roseaux tressés, au milieu d'enclos propres et soigneusement entretenus. Cette réunion d'une cinquantaine de cases occupait une légère dépression de terrain dans une vallée étendue entre de basses montagnes. La violence du vent portait plus avant qu'il ne convenait au docteur [...].

(Frantext: Jules Verne, Cinq semaines en ballon, Paris, Lidis, I957, p. I64)

[47] «Ils ont raison!» s’écrie Damayantî, cachée derrière un arbre, et, entendant ces menaces, elle se sauve comme se fuyant elle-même. «Je porte malheur à qui m'approche. Ma faute, laquelle? pour être en butte à tant de maux. Subirais-je la vengeance des dieux que j’ai refusé de choisir pour épouser Nala?» La fugitive arrive seule dans la ville de Tchédi. Les habitants prennent le frais sur les places publiques, ils considèrent avec étonnement cette femme moins qu'en haillons, marchant comme une insensée, les enfants la poursuivent et la huent.

(Frantext: Stéphane Mallarmé, Contes indiens, Toulouse, Éditions Ombres, 1993, p. 70) 
[48] Je traversai avec mon guide une partie de la place, et j'entrai chez Mme Nedling, grosse hollandaise fort gaie. Elle prenait le thé au milieu de sept ou huit officiers de la flotte, qui fumaient leur pipe. Elle me fit voir un appartement fort propre, et m'assura que tout ce qui était dans la maison était à mon service. Quand on a vu une ville hollandaise, on les a toutes vues: de même, chez les habitants, l'ordre d'une maison est celui de toutes les autres. Voici quelle était la police de celle de Mme Nedling. Il y avait toujours dans la salle de compagnie une table couverte de pêches, de melons, d'abricots, de raisins, de poires, de fromages, de beurre frais, de pain, de vin, de tabac et de pipes. À huit heures, on servait le thé et le café; à midi, un dîner très abondant en gibier et en poisson; à quatre heures, le thé et le café; à huit, un souper comme le dîner. Ces bonnes gens mangeaient toute la journée.

(Frantext: Henri Bernardin de Saint-Pierre, Voyage à l'T̂le de France, Paris, Ledentu, I840, p. 86-87)

On remarque alors certaines tendances dans la répartition des deux processus anaphoriques: continuité topicale plus fréquente avec le possessif et, corrélativement à cette continuité, continuité propositionnelle renforcée, par rapport au défini associatif. Le possessif est régulièrement utilisé lorsque la ville est particulièrement saillante (parce qu'elle a fait l'objet de plusieurs mentions, comme en [42] et [43], et/ou parce qu'elle est mentionnée en position de sujet, comme en [4I], [42] et [44]), et le défini associatif lorsqu'elle est beaucoup moins saillante (par exemple, si elle est mentionnée dans une position syntaxique qui confère peu de saillance, comme les compléments de lieu de [45] et [47], et qu'elle n'a pas fait l'objet de multiples mentions ${ }^{15}$ ). Mais, surtout, la cohésion propositionnelle se trouve régulièrement renforcée avec le possessif, de la même manière qu'elle l'est lorsqu'on préfère une reprise pronominale à une reprise par un nom propre (voir à cet égard les analyses de Kleiber [1994] et Schnedecker [1997], sur des séquences du type «Nom propre... Pronom personnel» vs «Nom propre... Nom propre»). Le possessif, parce qu'il opère une reprise coréférentielle et explicite le lien entre la collection et ses membres, apparait en effet comme un renfort de la cohésion propositionnelle et favorise alors l'expression de relations de cohérence plus cohésives, des relations causales. Cette plus grande force cohésive des relations causales, déjà observée, comme le rappelle Charolles (1995: 139), par David Hume dans ses Essais philosopbiques sur l'entendement bumain (1748), est confirmée par un certain nombre d'expériences psycholinguistiques. Knott et Sanders (1998: 138) évoquent à ce sujet des expériences montrant que les événements reliés par des relations causales sont mieux mémorisés que les autres ou encore que les séquences causales sont lues plus rapidement.

On a déjà pu souligner ces tendances avec d'autres relations associatives, telles que les relations méronymiques ou les relations fonctionnelles (voir Salles, 20I3

15. Le défini associatif est même parfois possible en l'absence de toute mention antécédente. C'est le cas dans le titre suivant: Gap: la passagère téléphone à la police car le conducteur est saoul (Le Post archives du 29 juin 20I0, en ligne: http://archives-lepost.huffingtonpost.fr/article/2010/o6/29/2134277_gap-lapassagere-appelle-la-police-car-le-conducteur-est-ivre.html), qui propose deux anaphoriques associatifs fonctionnels, la passagère et le conducteur, sans mention du véhicule. 
et 20I4), et on les observe dans nombre d'exemples ville-babitants. Alors que le possessif est régulièrement utilisé lors de l'expression de relations causales, le défini associatif apparaît souvent dans des contextes plus distendus, moins cohésifs. Les séquences [4I]-[44] se laissent toutes interpréter en termes de cause-conséquence : on peut à chaque fois, conformément à la définition de la relation cause-conséquence proposée par Cornish (2009a et b), inférer que l'état ou l'événement asserté via la phrase ou proposition qui précède l'occurrence du SN possessif, cause ou pourrait causer (voir la structure hypothétique de l'exemple [44]) l'état ou l'événement asserté via la phrase ou proposition qui comprend le SN possessif. Le massacre des habitants de l'exemple [4I], la jouissance du droit de cité romaine des habitants de l'exemple [42], le nouvel état de ceux de l'exemple [43] sont ou seront les conséquences, respectivement, de la prise de la ville par les ennemis, de ce que les Romains ont fait d'Antibes après la conquête des Gaules, de ce que Dieu fera de Jérusalem; la nature des rêves des habitants de l'exemple [44] est présentée comme la conséquence attendue de la purification de la ville.

Les séquences avec anaphore associative mettent en jeu des relations de cohérence différentes: en [45]-[47], la phrase qui mentionne la ville dans laquelle entrent ou sont entrés les personnages de [45] et [47] ou que les personnages de [46] survolent, fournit essentiellement le cadre spatio-temporel dans lequel l'événement ou l'état exprimé dans la phrase qui comprend l'anaphorique associatif les habitants se situe. L'exemple [45] pourrait autoriser une interprétation consécutive (Jacques et son maitre entrent dans la ville et sont alors assaillis par les habitants, qui quittent leurs maisons et se joignent à ceux qui poursuivaient déjà Jacques), si les temps verbaux utilisés étaient les mêmes (passé composé/passé composé: Ils sont entrés dans la ville. Les habitants ont [alors] quitté leurs maisons; ou présent/présent: Ils entrent dans la ville. Les habitants quittent [alors] leurs maisons), mais, ici, le passage du passé composé au présent n'autorise pas une telle lecture: ils sont déjà dans la ville quand les habitants se joignent aux autres. Le passé composé, avec le verbe perfectif entrer, insiste sur le résultat lié à l'achèvement du procès («ils sont dans la ville») et fournit ainsi le cadre dans lequel l'événement suivant a lieu. Les exemples [46] et [47] ne se prêtent pas non plus à une lecture consécutive: les événements ou états dénotés sont simplement concomitants, et la relation entre les deux est, comme en [45], de l'ordre de la circonstance:

Circonstance: Une proposition $\mathrm{P}^{\circ}$ exprimant un état, processus ou événement dans $\mathrm{S}^{\circ}$ sera comprise comme fournissant le cadre temporel, spatial ou cognitif à l'intérieur duquel l'événement dénoté par $\mathrm{S}^{\mathrm{t}}$ est à situer. L'événement principal (exprimé par $\mathrm{S}^{\mathrm{I}}$ ) devra être soit totalement inclus dans l'événement ou l'état circonstanciel $\left(\mathrm{S}^{\circ}\right)$ ou le chevaucher. (définition de Cornish, 2009b: I69, fondée sur celle de Mann et Thompson, 1988)

$\mathrm{S}^{\circ}$, qui mentionne l'arrivée dans la ville en [47] ou son survol en [46], fournit ici le cadre spatio-temporel dans lequel se situe l'état ou l'événement associé aux habitants (prendre le frais sur les places publiques, travailler à la récolte du coton). 
Le dernier exemple d'anaphore associative (en [48]) ne repose pas non plus sur une relation causale, mais sur une relation de ressemblance (voir Hobbs, 1990; Kehler, 2002 et 2004), avec l'analogie, explicitée par le connecteur de même, entre les villes hollandaises, qui se ressemblent toutes, et la vie domestique de leurs habitants, qui est toujours la même.

Avec les exemples [4I]-[48], on retrouve les trois principes de cohérence de Hobbs (1990), inspirés de Hume (Essais philosopbiques sur l'entendement bumain, I748), le plus cohésif, la causalité, étant associé à l'emploi du possessif:

- la causalité, avec la relation cause-conséquence et les anaphores possessives (exemples [4I]-[44]),

- la ressemblance, avec l'analogie et l'anaphore associative de l'exemple [48],

- la contiguïté (spatio-temporelle) ${ }^{16}$, avec la relation circonstance et les anaphores associatives des exemples [45]-[47].

\section{Conclusion}

Les fonctionnements anaphoriques spécifiques à la relation membre-collection reflètent à la fois les propriétés référentielles des collections (notamment leur homogénéité interne) et les propriétés sémantiques de la relation membre-collection (l'orientation de la collection vers les membres, et ainsi, le caractère généralement non relationnel du nom de membre).

L'homogénéité interne qui caractérise les collections explique pourquoi l'anaphore associative n'est pas possible avec certains noms, les noms génériques de membres (membre, élément):

[49] Le jury a enfin fini de délibérer. ?Les membres/Ses membres viennent de regagner leurs sièges.

En [49], l'emploi du nom membres met l'homogénéité de la collection en avant, la référence se fait de façon indifférenciée à l'ensemble des membres du jury. Or, l'anaphore associative réclame au contraire une différenciation des membres de la collection, ce que l'exemple [50], contrairement à l'exemple [49], permet:

[5०] Le jury a enfin fini de délibérer. Les membres titulaires / Le président et les (autres) jurés viennent de regagner leurs sièges.

Le caractère généralement non relationnel (catégorématique) du nom de membre explique pourquoi le possessif ne constitue pas un véritable concurrent au défini associatif dans les autres cas. Avec les noms catégorématiques, le possessif est le plus souvent utilisé pour référer à des antécédents humains. Seuls les noms relationnels

16. Ou figure-fond; voir Cornish, 2006, 2009a et b. 
(méronymes, noms fonctionnels) échappent à cette préférence pour un "possesseur» humain: la dépendance relationnelle (son tronc $=$ le tronc du tilleul, son conducteur $=$ le conducteur de la voiture) peut en effet facilement l'emporter sur une relation de dépendance contingente. En d'autres termes, c'est essentiellement avec les relations méronymiques et fonctionnelles qu'on observera une véritable concurrence entre anaphore associative et anaphore possessive, et non avec la relation membre-collection.

Dans les rares configurations réellement favorables aux deux déterminants (par exemple avec un nom relationnel comme babitants, qui peut être considéré comme un nom fonctionnel ou un nom de membres de ville, village, etc.), on retrouvera les mêmes tendances que celles observées avec les relations méronymiques et fonctionnelles: le possessif, en opérant une reprise coréférentielle et en explicitant le lien entre la collection et le membre, soutiendra fréquemment l'expression de relations de cohérence plus cohésives que celles qui seront généralement associées à l'emploi du défini associatif.

\section{Références}

Barker, C. 20II. Possessive and Relational Nouns. In K. von Heusinger, C. Maienborn et P. H. Portner (éd.), Semantics. An International Handbook of Natural Language Meaning. Berlin: De Gruyter Mouton. Vol. 2: IIo9-IIzo.

BARTNING, I. 1996. Éléments pour une typologie des SN complexes en de en français. Langue française 109: 29-43.

Borillo, A. 1997. Statut et mode d'interprétation des noms collectifs. In C. Guimier (éd.), Co-texte et calcul du sens. Caen: Presses universitaires de Caen: I05-I2I.

Charolles, M. 1995. Cohésion, cohérence et pertinence du discours. Travaux de linguistique 29: I25-I5I.

Cornish, F. 2006. Relations de cohérence et anaphores en contexte inter-phrastique : une symbiose parfaite. Langages $163: 37-55$.

Cornish, F. 2009a. Inter-sentential Anaphora and Coherence Relations in Discourse : A Perfect Match. Language Sciences 3I (5): 572-592.

Cornish, F. 2009b. Le rôle des anaphores dans la mise en place des relations de cohérence dans le discours: l'hypothèse de J. R. Hobbs. Journal of French Language Studies I9 (2): I59-I8I.

Flaux, N. 1998. Les noms collectifs et la prédication. In M. Forsgren, K. Jonasson et H. Kronning (éd.), Prédication, assertion, information (Actes du colloque d'Uppsala en linguistique française, 6-9 juin 1996). Uppsala: Acta Universitatis Upsaliensis : 173-183.

Flaux, N. 1999. À propos des noms collectifs. Revue de linguistique romane 63 (25I-252): 47I-502.

FlaUX, N. et VAN DE VELDE, D. 2000. Les noms en français: esquisse de classement. Gap - Paris: Ophrys.

Fraurud, K. 1992. Processing Noun Phrases in Natural Discourse. Thèse de doctorat non publiée. Université de Stockholm. 
Fraurud, K. 1996. Cognitive Ontology and NP Form. In T. Fretheim et J. K. Gundel (éd.), Reference and Referent Accessibility. Amsterdam - Philadelphie : J. Benjamins : 65-87.

Hawkins, R. 1981. Towards an Account of the Possessive Constructions: NP's $N$ and the $N$ of NP. Journal of Linguistics I7 (2): 247-269.

Herslund, M. 1996. Partitivité et possession inaliénable. Faits de langues 7: 33-42.

Hız, H. 1968. Referentials. In Transformations and Discourse Analysis Project 76. Philadelphie: Department of Linguistics, University of Pennsylvania: I-37. En ligne à l'adresse suivante: http://www.cs.nyu.edu/cs/projects/lsp/pubs/tdap_76_i968.pdf.

Hoвbs, J. R. 1990. Literature and Cognition. Stanford: CSLI Publications. Chap. 5: The Coherence and Structure of Discourse: 83-II4.

Joosten, F. 2oro. Collective Nouns, Aggregate Nouns, and Superordinates. When «Part of» and «Kind of» Meet. Lingvistice Investigationes 33 (I): $25-49$.

KeHler, A. 2002. Coherence, Reference, and the Theory of Grammar. Stanford: CSLI Publications.

Kehler, A. 2004. Discourse Coherence. In L. R. Horn et G. L. WARD (éd.), The Handbook of Pragmatics. Malden - Oxford: Blackwell: 24I-265.

KLeIBer, G. 1981. Problèmes de référence: descriptions définies et noms propres. Metz - Paris: Université de Metz - Klincksieck.

KLEIBER, G. 1994. Anaphores et pronoms. Louvain-la-Neuve: Duculot.

KLeibER, G. 1999. Les noms relationnels en anaphore associative: le cas des noms de parenté. Studi italiani di linguistica teorica e applicata 28 (2): 283-300.

KLeiber, G. 200o. Le possessif via l'anaphore associative. In M. L CASAl Silva et al. (éd.), La Lingüística francesa en España camino del siglo XXI. Madrid: Arrecife Producciones: 55-74. En ligne à l'adresse suivante: http://dialnet.unirioja.es/descarga/articulo/4044357. pdf [également publié en 200I. In L. TAsmowski (éd.), The Expression of Possession in Romance and German Languages. Cluj-Napoca: Editura Clusium: 29-52].

KLeIber, G. 20oia. L'anaphore associative. Paris: PUF.

Kleiber, G. 2ooib. Sur le chemin du comptable au massif. In C. Buridant, G. Kleiber et J.-C. Pellat (éd.), Par monts et par vaux: itinéraires linguistiques et grammaticaux. Mélanges offerts au professeur Martin Riegel. Louvain - Paris: Peeters: 219-234.

KLEIBER, G. 2003. Un «puzzle» référentiel en anaphore associative. In F. I. FonseCA et A. M. Brito (éd.), Lingua portuguesa: estruturas, usos e contrastes. Porto: Centro de Linguística da Universidade do Porto: 97-IIo.

Kleiber, G. 2004. Défini associatif et possessif en concurrence textuelle. In J.-M. AdAm, J.-B. Grize et M. Ali Bouacha (éd.), Texte et discours: catégories pour l'analyse (Actes du colloque tenu à l'université de Bourgogne du 5 au 7 juin 2002). Dijon : Éditions universitaires de Dijon: 5I-6I.

Kleiber, G. 2008. The Semantics and Pragmatics of the Possessive Determiner. In H. H. Müller et A. Klinge (éd.), Essays on Nominal Determination: From Morphology to Discourse Management. Amsterdam - Philadelphie: J. Benjamins: 309-336.

Kleiber, G. 2009. Défini et possessif anaphoriques. In F. Venier (éd.), Tra pragmatica e linguistica testuale. Ricordando Maria-Elisabeth Conte. Alexandrie (It.): Edizioni dell'Orso: 279-306. 
Kleiber, G. 20II. Sémantique et pragmatique du déterminant possessif. L'Information grammaticale I29: 3-I3.

Knott, A. et SAnders, T. 1998. The Classification of Coherence Relations and Their Linguistic Markers: An Exploration of Two Languages. Journal of Pragmatics 30 (2): I35-I75.

LAmmert, M. 2005. Les noms collectifs: entre quantification et qualification. Scolia 20: $24 \mathrm{I}^{-2} 58$

LAMMERT, M. 2006. Marqueurs de partition: outils métalinguistiques dans l'analyse des noms collectifs. In G. Kleiber, C. Schnedecker et A. Theissen (éd.), La relation partie-tout. Louvain - Paris - Dudley: Peeters: IO7-I22.

Lammert, M. 2oio. Sémantique et cognition. Les noms collectifs. Genève - Paris: Droz.

Lecolle, M. 1998. Noms collectifs et méronymie. Cabiers de grammaire 23: 4I-65.

LeIsI, E. 197I. Der Wortinbalt. Seine Struktur im Deutschen und Englischen. Heidelberg: Quelle und Meyer [4 éd.].

Mann, W. C. et Thompson, S. A. 1988. Rhetorical Structure Theory: Toward a Functional Theory of Text Organization. Text 8 (3): 243-28r. En ligne à l'adresse suivante: http:// www.cis.upenn.edu/ nenkova/Courses/cis7oo-2/rst.pdf.

Michaux, C. 1992. The Collectives in French: A Linguistic Investigation. Lingvistice Investigationes I6 (I): 99-I24.

Minatsch, W. 2000. La relation partie-tout aux confins de l'hyponymie. Scolia I2: 237-260.

Milner, J.-C. 1982. Ordres et raisons de langue. Paris: Seuil.

Reichler-BÉGuelin, M.-J. I988. Anaphore, cataphore et mémoire discursive. Pratiques 57 : I5-43.

SAlles, M. 20I3. Anaphore associative et anaphore possessive. Quelles différences pour les relations de cohérence? Revue romane 48 (I) : $5 \mathrm{I}^{-7} 78$.

SAlles, M. 20I4. Possessif ou défini associatif? Les relations fonctionnelles et actancielles. Studii de lingvistică 4: 199-215. En ligne à l'adresse suivante: http://studiidelingvistica. uoradea.ro/docs/4-20I4/articole pdf SL4/Salles.pdf.

Schnedecker, C. 1997. Nom propre et chaînes de référence. Metz - Paris: Université de Metz - Klincksieck

VILlar, M. B. 2006. Quel est le rôle de la possession inaliénable dans la description de la relation lexicale partie-tout? In G. Kleiber, C. Schnedecker et A. Theissen (éd.), La relation partie-tout. Louvain - Paris - Dudley: Peeters: $367-379$. 